\title{
EL COSTO DE LA VIDA EN LA GRECIA ANTIGUA
}

\author{
Roberto Morales Harley ${ }^{1}$
}

Recibido: 30/06/2014

Aprobado: 17/08/2014

\begin{abstract}
RESUMEN
El artículo se centra en los aspectos económicos más relevantes de la Antigua Grecia, más específicamente, de la Atenas del siglo V a.C. Explora la noción griega de economía, el sistema monetario, la administración financiera y el mundo laboral, con el propósito de contextualizar el costo de la vida. Los ejemplos al respecto toman en consideración los costos de los productos y los salarios de las personas.
\end{abstract}

PALABRAS CLAVE: ECONOMÍA, GRECIA ANTIGUA, COSTO DE LA VIDA, MONEDAS GRIEGAS

\section{ABSTRACT}

The paper focuses on the most relevant economic aspects of Ancient Greece, more specifically, 5th century BC Athens. It explores the Greek notion of economy, the monetary system, the financial administration and the labor market, in order to contextualize the cost of living. The examples on this matter take into account the products' costs and the people's wages.

KEY WORDS: ECONOMY, ANCIENT GREECE, COST OF LIVING, GREEK COINS

1 Universidad de Costa Rica, Escuela de Filología, Lingüística y Literatura, Código Postal 11501-2060, Costa Rica; roberto.moralesharley@gmail.com 


\section{INTRODUCCIÓN}

Economía es una palabra de origen griego, pues deriva de oĩ en la Grecia Antigua, su sentido se refiere, no tanto al manejo de bienes, objeto de la crematística, del griego $\chi \rho \eta ́ \mu \alpha \tau \alpha$, 'bienes'; sino, más bien, a la administración en el ámbito interno, en oposición al externo, que sería objeto de la política, del griego wó $1 \varsigma$, 'ciudad'. Tomando en cuenta estas ideas, vale la pena ponderar la definición y los significados actuales del término, desde una perspectiva etimológica, con el fin de destacar sus raíces griegas, al tiempo que se explican las diferencias.

El DRAE (Diccionario de la Real Academia Española, 2013) ofrece, para el concepto de economía, la siguiente información:

Economía. (Del lat. oeconomia, y este del gr. oikovouía). 1. f. Administración eficaz y razonable de los bienes. 2 . $\mathrm{f}$. Conjunto de bienes $y$ actividades que integran la riqueza de una colectividad o un individuo. 3. f. Ciencia que estudia los métodos más eficaces para satisfacer las necesidades humanas materiales, mediante el empleo de bienes escasos. 4. f. Contención o adecuada distribución de recursos materiales o expresivos. 5 . f. Ahorro de trabajo, tiempo o de otros bienes o servicios. 6. f. pl. Ahorros mantenidos en reserva. 7. f. pl. Reducción de gastos anunciados o previstos.

Todas las acepciones se relacionan con la idea de bienes, aunque desde diversas perspectivas: una práctica administrativa (1), el objeto de dicha práctica (2), su aspecto teórico (3), o, en los casos restantes (4-7), un uso particular con el sentido de 'reducción'.

Economía es una voz compuesta. Su primer miembro deriva del griego oĩ vivienda; habitación, cuarto; sala, comedor; templo; jaula, nido; residencia; bienes, propiedad, hacienda, fortuna; familia, linaje; servidumbre, criados; patria" (Pabón, 1999: 420). Este remite a un espacio familiar cerrado, que abarca tanto a las personas como sus posesio- nes. El segundo término procede del griego $v \varepsilon ́ \mu \omega$, "repartir, distribuir; asignar, atribuir; conceder, permitir, admitir; respetar, observar; dividir, partir; apacentar, llevar a pacer; consumir, devorar, devastar, asolar, destruir; poseer, tener, ocupar, habitar; dirigir, conducir, administrar, manejar, tener por, considerar como" (Pabón, 1999: 408). La noción relevante aquí es la de 'administración'.

El griego oíкоvouía, "administración de una casa", pasa al latín oeconomia, con el sentido de "organización, disposición, administración, economía”, y al español economía, atestiguado por primera vez en 1607 (Segura, 2003: 513). ¿Cómo se llegó de la casa al estado? El punto de contacto es el oĩkos, la estructura social fundamental del mundo griego antiguo. El concepto es polisémico:

En primer lugar, oikos significa "casa" en el sentido más literal y material, es decir, como vivienda, el espacio físico donde habita la unidad social básica, $y$ pudo ser el significado original de la palabra... El segundo significado de oikos es propiedades, que abarcan tanto bienes inmuebles como muebles, tanto inanimados como animados... Por último, oikos también sirve para hacer referencia específica a sus componentes humanos libres, es decir, a la primera y básica sociedad humana: la asociación de hombre y mujer y el producto de dicha asociación, los hijos (Mirón, 2004: 62-63).

Entre las definiciones griegas de economía, destacan la de Jenofonte y la del PseudoAristóteles. Para el primero, "la administración de la casa es el nombre de un saber" (Jenofonte, 2006: 1); para el segundo, "un saber que tiene por objeto la adquisición y uso del oikos" (1343a5-9). Mirón (2004: 66), señala la oposi-

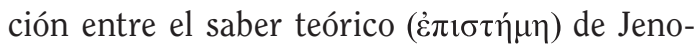
fonte $y$ el saber práctico ( $\tau \dot{\varepsilon} \chi \vee \eta)$ del PseudoAristóteles, ambas nociones conservadas en las acepciones del DRAE.

La economía, como administración de la casa, es anterior a la política, como admi- 
nistración de la ciudad. Mientras que aquella se remonta a la época de Homero (siglo VIII a.C.), esta alcanza su punto más alto en época de Pericles (siglo V a.C.), si bien las primeras ciudades son anteriores. En griego, $\pi$ $\lambda \iota_{\iota} \varsigma$ es la ciudad-estado, y su administra-

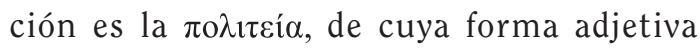

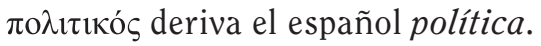

El modo de vida de la polis se basa en la autarquía, autosuficiencia ejemplificada en una tierra que ofrece productos, un comercio que genera ganancias, unas tropas que permiten la defensa contra los vecinos (Burckhardt, 1974: 105). Los ciudadanos deben conocerse entre sí, por lo que su número no debe ser muy elevado: una ciudad ideal es aquella que no pasa de 10000 habitantes. En este contexto, las relaciones comerciales se establecen entre una polis y otra, lo que resulta en la implementación de un sistema monetario.

\section{PRESENTACIÓN DE DATOS}

\section{Sistema monetario}

Para hallar el origen del sistema monetario griego, es preciso acudir al sistema de pesos. Procedentes del mundo oriental, antes de Solón, coexistían tres sistemas: el egineta, de origen fenicio, que llegó a través de las relaciones comerciales con la isla de Egina; el euboico, copia de un sistema persa, propagado por los mercaderes eubeos de Calcis y Eritrea; $y$ el antiguo sistema ático. El sistema euboico se usaba para el peso de las monedas; pero, para las transacciones comerciales, se mantuvo el egineta. En todos ellos, la unidad básica de peso es el talento ( $\tau \alpha \dot{\lambda} \alpha \nu \tau \tau o v)$, que significa "platillo de la balanza" (Pabón, 1999: 573) y equivale al peso de un pie cúbico de agua. Según la información de Bailly (2000: 2197), ofrecemos el siguiente cuadro con las correspondencias de los pesos en los tres sistemas:

CUADRO 1

SISTEMAS DE PESOS GRIEGOS ANTES DE SOLÓN

\begin{tabular}{lll}
\hline Sistema egineta: & Sistema euboico: & Antiguo sistema ático: \\
\hline 1 talento $=37,11 \mathrm{~kg}$ & 1 talento $=25,92 \mathrm{~kg}$ & 1 talento $=35,937 \mathrm{~kg}$ \\
$1 \mathrm{mina}=618 \mathrm{gr}$ & 1 mina $=432 \mathrm{gr}$ & $1 \mathrm{mina}=0,599 \mathrm{gr}$ \\
$1 \mathrm{dracma}=6,16 \mathrm{gr}$ & 1 dracma $=4,32 \mathrm{gr}$ & 1 dracma $=0,006 \mathrm{gr}$ \\
\hline
\end{tabular}

Fuente: Bailly (2000: 2197).

Con Solón, el sistema de pesos se unifica, si bien subsiste una diferencia entre el peso monetario (peso físico de las monedas, calculado según el sistema euboico) y el mercantil (valor de intercambio de las monedas en el mercado, basado en el sistema egineta). Se añaden nuevas unidades de peso: el calco, deri- vado de $\chi \alpha \lambda$ кós, "cobre, bronce" (Pabón, 1999: 638); el hemióbolo, o medio óbolo; y el óbolo, que significa "broche", pues en tiempos remotos los broches de hierro sirvieron como medio de cambio (Flacelière, 1956: 137). Igualmente, nos basamos en Bailly (2000: 2197) para las correspondencias: 
CUADRO 2

SISTEMA DE PESOS GRIEGO DESPUÉS DE SOLÓN

\begin{tabular}{|c|c|c|}
\hline Unidad & Peso monetario & Peso mercantil \\
\hline 1 calco $\left(\chi \alpha \lambda_{\kappa o v ̃} \varsigma\right)$ & $0,09 \mathrm{gr}$ & - \\
\hline 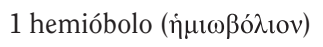 & $0,36 \mathrm{gr}$ & - \\
\hline 1 óbolo (ỏßo & $0,72 \mathrm{gr}$ & $1 \mathrm{gr}$ \\
\hline 1 dracma ( $\left.\delta \rho \alpha \chi \mu)^{\prime}\right)$ & $4,32 \mathrm{gr}$ & $6 \mathrm{gr}$ \\
\hline $1 \operatorname{mina}(\mu v \tilde{\alpha})$ & $432 \mathrm{gr}$ & $606 \mathrm{gr}$ \\
\hline 1 talento ( $\tau \dot{\alpha} \lambda \alpha \nu \tau \tau o v)$ & $25,92 \mathrm{~kg}$ & $36,39 \mathrm{~kg}$ \\
\hline
\end{tabular}

Fuente: Bailly (2000: 2197).

Este sistema se mantiene hasta época helenística. La siguiente reforma le corresponderá a los ptolomeos, con el propósito de hacer coincidir las medidas con las egipcias. Su modelo acaba también por extenderse hasta el Ática, gracias al comercio.

Con los valores del sistema de peso en mente, se puede proceder a las monedas. La palabra moneda procede el latín Moneta, "sobrenombre de la diosa Juno, como correspondiente a su advocación como 'consejera'; junto a su templo se acuñaba la moneda" (Segura, 2003: 471). En el 388 a.C., los galos se aprestaban a tomar la colina del Capitolio en Roma, cuando los gansos, ave representativa de la diosa, despertaron a los soldados para que pudieran salvar la ciudad. De la acción de 'advertir', en latín moneo, deriva el apelativo de Moneta, 'la que advierte'. En griego, la palabra es vó $\mu 1 \sigma \mu \alpha$, "uso, costumbre, regla; moneda, dinero; institución” (Pabón, 1999: 412), de donde viene numismática. Las primeras monedas griegas proceden de Asia menor $y$ se remontan al siglo VII a.C.

The first coins were cast in Lydia (in western Turkey) in the late seventh century, but the Greeks leaped on this new medium in the early sixth. The earliest issues probably had more political than economic functions, since they were for very large denominations, and made of electrum, a naturally occurring alloy of gold and silver, which made it hard to judge their metal value. By the late sixth century, though, small denomination bronze coins were circulating, which must mean that exchange was already becoming monetized (Morris, 2004: 731).

Las primeras monedas fueron fundidas en Lidia (en Turquía occidental) a fines del siglo sétimo, pero los griegos dieron el salto hacia este nuevo medio a inicios del sexto. Las primeras emisiones probablemente tenían funciones más políticas que económicas, dado que eran por denominaciones muy grandes $y$ hechas de electro, una aleación natural de oro y plata, la cual hacía difícil juzgar su valor metálico. Pero, para fines del siglo sexto, circulaban monedas de bronce con denominaciones pequeñas, lo cual debe significar que el intercambio ya se había vuelto monetario (Traducción del autor).

Cada estado solía acuñar su propia moneda, con su denominación y su emblema. Habitualmente, estos incluían representaciones de dioses, de animales, de plantas. De acuerdo con Flacelière (1956: 138), en Atenas, las monedas tenían, en el reverso, la cabeza de Atenea, con un casco y una corona de laurel, $y$, en el anverso, la lechuza de Atenea, con una medialuna, un gajo de olivo y las primeras tres letras del nombre de la ciudad. Las monedas se llamaban "lechuzas del Laurio" y existía el proverbio "es inútil llevar lechuzas a Atenas". Empero, existía también un sistema de pesos generalmente aceptado, mediante el cual se fijaba el valor relativo dentro de cada sistema. Con el paso del tiempo, esto evolucionó hacia un sistema de acuñación (Petrie, 1978: 108). 
Las principales monedas acuñadas en Atenas fueron el óbolo, el dracma, el didracma $y$ el tetradracma. En el siglo V a.C., las monedas usadas eran de plata; en el IV a.C., se introducen de bronce, las cuales duran poco tiempo en circulación, $y$ de oro, principalmente de origen extranjero. La nomenclatura también deriva del sistema de pesos. La unidad monetaria es el dracma de 4,31 gr o 4,32 gr. La menor denominación era el octavo de óbolo; la mayor, el estátero de oro. A partir de datos hallados principalmente en Bailly (2000: 2199) y Morkholm (1982, 290), se presentan estos cuadros sinópticos:

CUADRO 3

MONEDAS DE COBRE

\begin{tabular}{|c|c|c|c|}
\hline Unidad & Relación & Peso & Valor $^{2}$ \\
\hline 1 calco ( $\chi \alpha \lambda \kappa о \tilde{\nu} \varsigma)$ & 1/8 óbolo & $0,09 \mathrm{gr}$ & 0,1 dólares \\
\hline 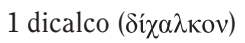 & 1/4 óbolo & $0,18 \mathrm{gr}$ & 0,2 dólares \\
\hline
\end{tabular}

Fuente: Elaboración propia a partir de Bailly (2000: 2199) y Morkholm (1982: 290).

CUADRO 4

MONEDAS DE PLATA

\begin{tabular}{|c|c|c|c|}
\hline Unidad & Relación & Peso & Valor \\
\hline $\begin{array}{l}1 \text { hemióbolo } \\
\left(\dot{\eta} \mu 1 \omega \beta \lambda_{\lambda} \text { tov }^{2}\right.\end{array}$ & 1/2 óbolo & $0,36 \mathrm{gr}$ & 0,4 dólares \\
\hline $\begin{array}{l}1 \text { óbolo } \\
\text { (óßo }{ }^{\prime} \varsigma \text { ) }\end{array}$ & $1 / 6$ dracma & $0,72 \mathrm{gr}$ & 0,8 dólares \\
\hline 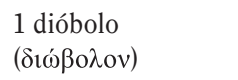 & $1 / 3$ dracma & $1,44 \mathrm{gr}$ & 1,6 dólares \\
\hline 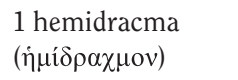 & $1 / 2$ dracma & $2,16 \mathrm{gr}$ & 2,4 dólares \\
\hline 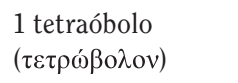 & $2 / 3$ dracma & $2,88 \mathrm{gr}$ & 3,2 dólares \\
\hline $\begin{array}{l}1 \text { dracma } \\
(\delta \rho \alpha \chi \mu \eta \dot{)})\end{array}$ & $1 / 100$ mina & $4,32 \mathrm{gr}$ & 4,8 dólares \\
\hline 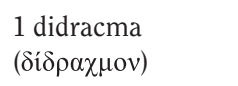 & $1 / 50$ mina & $8,64 \mathrm{gr}$ & 9,6 dólares \\
\hline 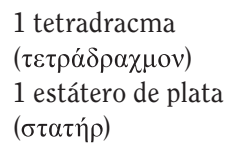 & $1 / 25$ mina & $17,28 \mathrm{gr}$ & 19,2 dólares \\
\hline $\begin{array}{l}1 \text { decadracma } \\
(\delta \varepsilon \kappa \alpha ́ \delta \rho \alpha \chi \mu o v)\end{array}$ & $1 / 10$ mina & $43,2 \mathrm{gr}$ & 48 dólares \\
\hline
\end{tabular}

Fuente: Elaboración propia a partir de Bailly (2000: 2199) y Morkholm (1982: 290).

$2 \quad$ Bailly (2000) ofrece el valor aproximado de cada moneda en francos de oro, según su valor en 1894, año de la primera edición del diccionario. Con el convertidor del Instituto Nacional de Estadística y Estudios Económicos de Francia (2013), el cual determina una equivalencia del poder adquisitivo para una determinada suma de dinero de un año a otro, tomando en cuenta la inflación, hemos podido expresar, si bien de manera aproximada, este valor en dólares, según su valor actual (todas las cifras en dólares que aparecen en el artículo corresponden al año 2013). Así, 1 franco de oro = 5,14 dólares. Lamentablemente, Bailly no especifica el método empleado para estimar sus equivalencias, por lo que los valores extraídos a partir de ellas deben considerarse en términos relativos (ingresosgastos) y a modo de ilustración. 
CUADRO 5

MONEDAS DE ORO

\begin{tabular}{|c|c|c|c|}
\hline Unidad & Relación & Peso & Valor \\
\hline 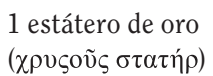 & $1 / 5$ mina & $86,4 \mathrm{gr}$ & 96 dólares \\
\hline
\end{tabular}

Fuente: Elaboración propia a partir de Bailly (2000: 2199) y Morkholm (1982: 290)

CUADRO 6

UNIDADES DE CÓMPUTO SIN EQUIVALENTE MONETARIO

\begin{tabular}{|c|c|c|c|}
\hline Unidad & Relación & Peso & Valor \\
\hline $\begin{array}{l}1 \text { mina } \\
(\mu \nu \tilde{\alpha})\end{array}$ & $1 / 60$ talento de plata & - & 480 dólares \\
\hline $\begin{array}{l}1 \text { talento de plata } \\
(\tau \alpha \dot{\alpha} \lambda \alpha \nu \tau o v)\end{array}$ & $1 / 10$ talento de oro & - & 28.800 dólares \\
\hline 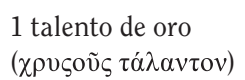 & - & - & 288.000 dólares \\
\hline
\end{tabular}

Fuente: Elaboración propia a partir de Bailly (2000: 2199) y Morkholm (1982: 290)

Adicionalmente, hubo otras denominaciones. El estátero de Alejandro, en oro, equivale al "filipo" macedonio y al "darío" persa (4 dracmas). El estátero de Cícico, en electro (aleación de 4 partes de oro y 1 de plata), tenía un valor mayor ( 28 dracmas).

El precio se decía en caso genitivo, que en la gramática griega corresponde a un complemento de nombre, en este caso, de un sustantivo. Así, para expresar "el esclavo cuesta 5 minas" se utiliza la construcción $\delta o v ̃ \lambda o \varsigma \pi \varepsilon ́ v \tau \varepsilon$ $\mu \nu \tilde{\omega} v$, literalmente, "el esclavo (es) de 5 cinco minas (de precio)" (berenguer, 2002: 194).

El interés se calculaba por minas por mes. Por ejemplo, por 1 dracma, se pagaba un $12 \%$ de interés anual: 1 dracma es la centésima parte de 1 mina ( 1 mina $=100$ dracmas), entonces, por 1 dracma, el interés mensual es de $1 \%$, y el anual ( 1 años = 12 meses), de 12 $\%$; por 9 óbolos, se pagaba un $18 \%$, dado que 9 óbolos equivalen a 1,5 dracmas $(1$ dracma $=$ 6 óbolos). Una tasa de interés común oscilaba entre $12 \%$ y $18 \%$ (petrie, 1978: 108). En el siglo $v$ a.C., Aparecen el préstamo con interés $y$ los primeros banqueros, que eran metecos (extranjeros, establecidos en atenas, sin derecho de ciudadanía) o libertos (antiguos esclavos, a los que se les ha concedido su libertad). Fue este un periodo de gran actividad económica, especialmente a nivel del estado.

\section{Administración financiera}

La administración financiera dependía de tres entidades: el Consejo, encargado de la distribución de fondos; las comisiones subordina-

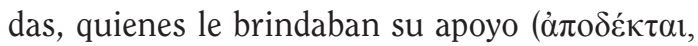

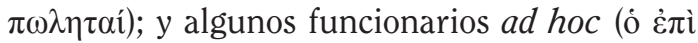

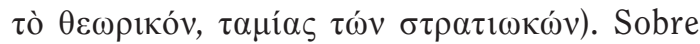
la base de los datos de Petrie (1978, 98-100), se incluyen algunos ejemplos de ingresos y egresos estatales.

\section{Ingresos:}

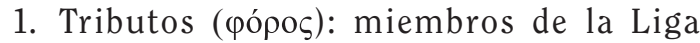
Ateniense (conocida como la Confederación de Delos, se conformó como resultado de las Guerras Médicas, con ciudades jonias y eolias de Asia Menor, ciudades 
de la Propóntide y de Tracia, los estados insulares de Lesbos, Quíos, Samos y varias de las islas Cícladas, así como la isla de Eubea). E.g., hacia 477 a.C., Arístides lo fijó en 460 talentos (13.248.000 dólares) anuales para el conjunto de miembros de la liga; hacia 445 a.C., Pericles, lo aumentó a 600 (17.280.000 dólares), de un total de 1.000 talentos (28.800.000 dólares) anuales que constituían las rentas públicas (Petrie, 1978: 35-43). Así, los tributos representan más de la mitad de los ingresos.

2. Impuestos: $2 \%$ sobre importaciones y expor-

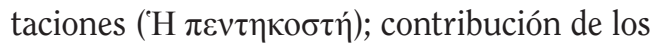
residentes extranjeros ( chos de bahía, de mercado, de tribunales, de las minas de plata en Laurio; impuesto

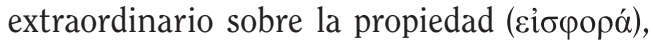
destinado a emergencias de guerra. E.g., para la sublevación de Mitilene, produjo 200 talentos (5.760.000 dólares). Para la recolección de impuestos los ciudadanos se dividían en compañías ( $\sigma v \mu \mu o p i ́ a 1)$, según su patrimonio; posteriormente, se eligió un cuerpo de 300 ciudadanos ricos que debían pagar por adelantado.

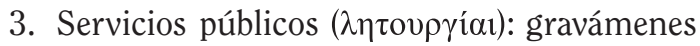
impuestos a ciudadanos pudientes, a título de prestación patriótica. Eran las siguientes: a) obligación de formar, equipar y adiestrar un coro para los festivales dramáticos

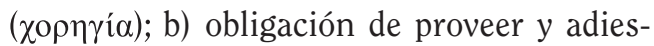
trar un grupo para las carreras de antorchas en los festivales religiosos ( $\left.\gamma v \mu v \alpha \sigma i \alpha \rho \chi^{\prime} \alpha\right)$; c) obligación de dirigir una embajada sacra a un festival religioso fuera de Ática

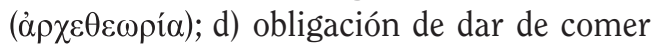
a los representantes de las tribus en ciertas

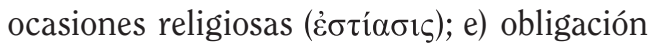
de preparar y mantener por un año un trirreme para el servicio público (

\section{Egresos:}

1. Salarios: funcionarios públicos.

2. Honorarios: miembros del Consejo, participantes en la Asamblea, jueces.

3. Servicios municipales: manutención de esclavos públicos.
4. Actividades culturales: festivales religiosos, competiciones dramáticas, musicales y atléticas. E.g., el Fondo Teórico pagaba la entrada al teatro para los ciudadanos más pobres, cuyo costo originalmente era de 2 óbolos (1,6 dólares).

5. Ejército: soldados. E.g., el sitio de Potidea tuvo un costo de 2.000 talentos (57.600.000 dólares).

\section{Mundo laboral}

En la épica de Homero, existe una visión harto negativa del trabajo, como se puede apreciar en la respuesta de Aquiles a Odiseo: "No pretendas, Ulises preclaro, buscarme consuelos / de la muerte, que yo más querría ser siervo en el campo / de cualquier labrador sin caudal y de corta despensa / que reinar sobre todos los muertos que allá fenecieron" (Od. XI, 488-491, Homero, 2002: 279). Tal estado de cosas mejora con Hesíodo, quien señala a su hermano Perses: "El trabajo no es ninguna deshonra; la inactividad es una deshonra" (Tr. 311, Hesíodo, 1997: 141).

Este desprecio por el trabajo se aprecia en el plano léxico: ßávavбos, en la Atenas del siglo $\mathrm{V}$ a. C., es el obrero del fuego (metalurgia, alfarería) y, por extensión, todo trabajador

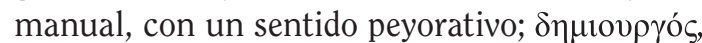
también en dicha Atenas, es el trabajador público, tanto el obrero como el intelectual, similar a lo que posteriormente se conoce como profesiones liberales, como los médicos. Los oficios manuales constituían actividades propias de esclavos y metecos, no de hombres libres (Flacelière, 1956: 136-137).

La agricultura es objeto de elogio en Jenofonte, cuyo himno a esta arte concluye así: "Estuvo muy acertado el que dijo que la agricultura era la madre y la nodriza de las demás artes, pues si la agricultura florece, prosperan también las otras artes, pero cuando la tierra se ve obligada a mantenerse yerma, se marchitan casi sin excepción las restantes artes, tanto en la tierra como en el mar" (Econ. V, 17). En la Grecia Antigua, los principales productos eran trigo, cebada, aceitunas, uvas, coles, lentejas, guisantes, cebollas y ajos. En la ganadería, destacan puercos, cabras, ovejas, además de asnos 
y mulos para el transporte. La lana se utilizaba para la elaboración de ropa.

La mayoría de la producción era para el propio consumo; sin embargo, paulatinamente se fueron generalizando las ventas, sobre todo de pan y de ropa. La economía poseía rasgos de industria, pero no había fábricas (Flacelière, 1956: 146). El trabajo no estaba reglamentado por el Estado, sino que dependía de la oferta y la demanda. Los contratos laborales se hacían de manera anual y se renovaban el 16 del mes de Antestérion (febrero). Se trabajaba en jornadas bastante extensas: desde la salida hasta la puesta del sol. Pero había 60 días feriados al año. En Flacelière (1956: 148-153), se encuentran varios ejemplos de oficios de obreros.

1. Minero ( $\mu \varepsilon \tau \alpha \lambda \lambda \varepsilon v \tau \eta ́ \varsigma)$ : llevaba una lámpara de aceite, pico, martillo, cincel, cestos. Recogía plomo y plata.

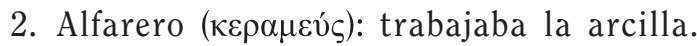
Creaba tinajas de barro cocido para almace-

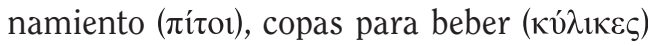
$y$ ollas ( $\chi v \dot{\tau} \rho \alpha \mathrm{l})$. Firmaba su obra.

3. Carpintero ( $\tau \dot{\varepsilon} \kappa \tau \omega v)$ : utilizaba escuadras, compases, objetos curvos para medir.

4. Curtidor ( $\sigma \kappa v \tau o \delta \varepsilon \psi o ́ \varsigma)$ o talabartero $(\beta v \rho \sigma o \delta \varepsilon ́ \psi \eta \varsigma)$ : trabaja el cuero antes que el zapatero.

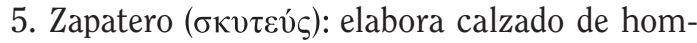
bre $y$ de mujer.

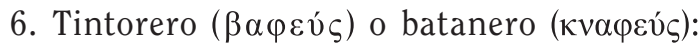
tiñe telas.

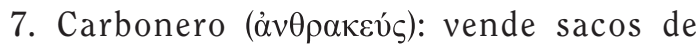
carbón.

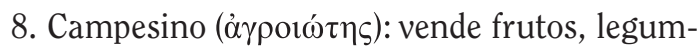
bres, quesos, aceite, vino.

9. Metalúrgico ( $\chi \alpha \lambda \kappa \varepsilon ́ v \varsigma)$ : trabaja cobre o bronce.

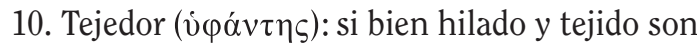
obras propias de mujeres.

Atenas fue un importante núcleo comercial, solamente superado después por Alejadría $y$ Rodas. Atenas es una región agradable y productiva: la tierra ofrece aceitunas, miel, trigo, higos; las canteras del Himeto y del Pentelicón, mármol; las minas de Laurión, plata. Sin embargo, esto no bastaba para alimentar a sus
500.000 habitantes, por lo que el principal sustento alimentario provenía de África: 800.0001.000.000 medidas de trigo al año (Burckhardt, 1963: 247). Esto significó un gran desarrollo del comercio de trigo, que estuvo siempre legislado de manera severa. Un comerciante de trigo

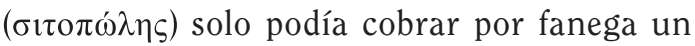
óbolo adicional al costo. Había comerciantes al por mayor ( $(\varepsilon \mu \pi \circ \rho \circ)$, como los de trigo, $y$ al por

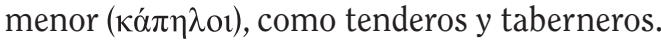

Con Pericles, Atenas alcanza el punto más alto de su hegemonía. Con el dominio del Egeo, disminuye el número de piratas y se desarrolla el comercio marítimo. Los barcos mercantes podían transportar hasta 400 toneladas, si bien la ausencia de mapas, brújulas y faros dificultaba la navegación. En esta época, los atenienses obtienen riquezas con su trabajo, pagan altos impuestos por sus bienes, ayudan a sus amigos necesitados $y$, de este modo, conservan su buena opinión (Burckhardt, 1963: 269).

En la ciudad, existen dos núcleos económicos: el Ágora y el Pireo. La primera es una especie de plaza del mercado, derivada del verbo

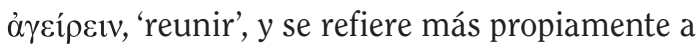
la acción de reunirse en asamblea que al lugar de reunión (Burckhardt, 1974: 101). Deambular por el ágora es una actividad común, cuya meta es negociar, pero también conversar y matar el tiempo. El Pireo es el puerto, "es el centro de una actividad mucho más importante que el Ágora, en la medida en que el comercio marítimo de Atenas supera su comercio terrestre" (Flaceliere, 1956: 156). En Atenas, el impuesto de aduanas fue de $1-50 \%$. A través del Pireo, ingresaba el trigo para la ciudad y el ejército.

Al igual que sucede con las finanzas públicas, los datos acerca de los costos de productos y los salarios de las personas son escasos. Sobre este punto, escribe Morris (2004: 709): "There are only scattered data on wages and hardly any long series of prices" (Solo hay datos aislados sobre los salarios y casi ninguna lista extensa de precios). Hay más información del siglo IV a.C. que del V a.C. Y se ha propuesto un crecimiento económico que va desde el siglo VIII a.C. hasta el III a.C., el cual se puede constatar, entre otras cosas, por el costo promedio de una vivienda. También es Morris (2004: 720) 
quien señala: "By 300, the typical Greek house cost something like five to ten times as much as the typical house had around 800 . It was bigger, better built, better drained, and had better protection against the elements" (Para el año 300, la típica casa griega cuesta algo así como cinco o diez veces lo que una casa típica cerca del año 800. Era más grande, mejor construida, mejor drenada y tenía un mejor protección contra la naturaleza).

Los datos sobre la calidad de vida, obtenidos no solo de fuentes literarias, sino también arqueológicas, conforman una base más sólida para el desarrollo de teorías económicas sobre el mundo antiguo. Esta es una tendencia relativamente reciente:

However, since the 1980s modern economic historians have generally moved away from earlier attempts to calculate changes in the real wage, concluding that there was too much variation between individual trades and towns to make the exercise very useful, and also recognizing that even tiny changes in the weighting of variables transformed the outcome... In the 1990s they undertook studies of discrete indices of wellbeing, such as stature, nutrition, mortality, morbidity, housing, and clothing (Morris, 2004: 713).

Sin embargo, desde los años 80 los historiadores económicos modernos generalmente se han alejado de los intentos previos de calcular cambios en el salario real, $y$ han concluido que hay mucha variación entre los oficios individuales $y$ los pueblos como para que el ejercicio sea de utilidad; asimismo, han reconocido que incluso pequeños cambios en la ponderación de las variables transformaba el resultado... En los años 90 emprendieron estudios de índices discretos de bienestar tales como estatura, nutrición, mortalidad, enfermedad, vivienda $y$ vestido (Traducción del autor).

Esto no quiere decir que no se pueda pintar un panorama, somero eso sí, del costo de la vida en la Atenas del siglo V a.C.

\section{Costo de la vida}

Amemiya (2007) ofrece la documentación más completa, en la bibliografía consultada, sobre la economía de la Grecia Antigua, del siglo V a.C. y especialmente del siglo IV a.C. A partir de su minuciosa recopilación de datos (Amemiya, 2007: 68-74), proponemos tres cuadros, los cuales proporcionan una ejemplificación del costo de la vida en la Atenas de Pericles. El primero presenta productos provenientes de diversos ámbitos (vivienda, vestido, alimentación, mobiliario), con sus respectivos costos (tanto en moneda griega como en su equivalente moderno aproximado); el segundo, distintos oficios remunerados, igualmente con la retribución monetaria correspondiente; $y$ el tercero, los valores promedios del salario, que fue evolucionando a lo largo del siglo. 


\section{CUADRO 7}

PRECIO DE PRODUCTOS EN LA ATENAS DEL SIGLO V A.C.

\begin{tabular}{ll}
\hline Producto & Precio \\
\hline 1 casa (vivienda) & 410 dracmas $=1.968$ dólares \\
1 cerdo (alimentación) & $40-20$ dracmas $=192-96$ dólares \\
1 cofre (mobiliario) & 21 dracmas $=100,8$ dólares \\
1 manto de lana (vestido) & 20 dracmas $=96$ dólares \\
1 piel de buey (mobiliario) & $8-6$ dracmas $=38,4-28,8$ dólares \\
1 sillón (mobiliario) & $8-6$ dracmas $=38,4-28,8$ dólares \\
1 fanega ${ }^{3}$ de trigo (alimentación) & 6 dracmas $=28,8$ dólares \\
1 silla (mobiliario) & $6-2$ dracmas $=28,8-9,6$ dólares \\
1 banco (mobiliario) & $5-1$ dracmas $=24-4,8$ dólares \\
1 mesa (mobiliario) & 4 dracmas $=19,2$ dólares \\
1 cochinillo (alimentación) & 3 dracmas $=14,4$ dólares \\
1 anguila de Beocia (alimentación) & 3 dracmas $=14,4$ dólares \\
1 fanega de olivas (alimentación) & 2 dracmas $=9,6$ dólares \\
1 congio de vino de Quíos (alimentación) & 2 dracmas $=9,6$ dólares \\
1 congio de vino ordinario (alimentación) & 4 óbolos $=3,2$ dólares \\
1 ánfora pintada (mobiliario) & 3 óbolos $=2,4$ dólares \\
1 cama individual (mobiliario) & 2 óbolos $=1,6$ dólares \\
1 mesa de noche (mobiliario) & 1 óbolo $=0,8$ dólares \\
\hline
\end{tabular}

Fuente: Elaboración propia a partir de (Amemiya, 2007: 68-74).

CUADRO 8

SALARIO POR JORNADA DE PERSONAS EN LA ATENAS DEL SIGLO V A.C.

\begin{tabular}{ll}
\hline Servicio & Salario \\
\hline 1 día de hoplita (soldado de infantería) & 2 dracmas $=9,6$ dólares \\
1 día de contador del Erecteón & $1,5-1$ dracmas $=7,2-4,8$ dólares \\
1 día de fiscal & 1 dracma $=4,8$ dólares \\
1 día de funcionario administrativo & 4 óbolos $=3,2$ dólares \\
1 sesión de la Corte & 2 óbolos $=1,6$ dólares \\
1 sesión de la Asamblea & 1 óbolo $=0,8$ dólares \\
\hline
\end{tabular}

Fuente: Elaboración propia a partir de (Amemiya, 2007: 68-74).

3 Es una unidad de volumen. Para comprender el cuadro, resultan necesarias las siguientes medidas: fanega

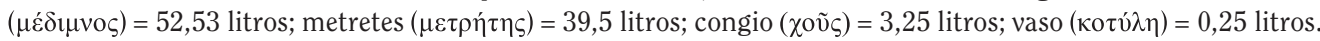


CUADRO 9

EVOLUCIÓN DEL SALARIO PROMEDIO EN LA ATENAS DEL SIGLO V A.C.

\begin{tabular}{ll}
\hline Periodo & Salario promedio \\
\hline $500-432$ & 4 óbolos por día (96 dólares por mes) \\
$432-412$ & 1 dracma por día (144 dólares por mes) \\
$412-400$ & Depende del trabajo \\
\hline
\end{tabular}

Fuente: Elaboración propia a partir de (Amemiya, 2007: 68-74).

Los precios son elevados con respecto a los salarios, particularmente para la dieta. La comida diaria usual incluía un trozo de pan, dos cebollas, tres aceitunas. Amemiya (2007: 76) hace una propuesta del costo diario de la vida de una familia promedio de cuatro integrantes, en época de Pericles (461-429): 1,76 óbolos (1,4 dólares) en trigo; 1,64 óbolos (1,3 dólares) en cebada; 0,5 óbolos ( 0,4 dólares) en miel; 0,0625 óbolos ( 0,05 dólares) en aceite de oliva; 0,5 óbolos (0,4 dólares) en vino; 0,2 óbolos ( 0,16 dólares) en legumbres; 1 óbolo (0,8 dólares) en companaje; para un total de 4,0225 óbolos $(3,22$ dólares). De acuerdo con estos datos, el costo de la vida resultaba excesivamente alto en la Atenas del siglo $\mathrm{V}$ a.C., dado que solo en alimentación de una familia promedio se podía gastar un poco más de un salario mínimo, el cual antes del 432 era de 4 óbolos (Amemiya, 2007: 72). Los salarios alcanzaban para un obrero soltero, pero no para una familia.

Al computar otros costos (Amemiya, 2007: 77-78), por ejemplo, 15 dracmas (72 dólares) al año en ropa y zapatos, y 36 dracmas (172,8 dólares) al año en alquiler, el monto total asciende a 307 dracmas (1.473,6 dólares) al año, de los cuales un $80 \%$ corresponde únicamente a la alimentación. Sin embargo, ya con el aumento salarial, después del 432, el monto promedio alcanza, al menos, para satisfacer las necesidades básicas.

\section{CONCLUSIONES}

Indudablemente, Atenas en el siglo V a.C. fue una ciudad floreciente. En este contexto, destacan grandes aportes para el mundo Occidental en los más diversos ámbitos del conocimiento: política, filosofía, historia, medicina, arquitectura, ingeniería, pintura, escultura, literatura, milicia, diplomacia, derecho, matemática, educación, entre otros. La economía en la Grecia Antigua es uno de estos temas fundamentales para comprender el desarrollo de aquella civilización; no obstante, los datos al respecto son escasos. Ante este panorama, la única respuesta posible para continuar profundizando en el estudio parece ser la interdisciplinaria. Por tal motivo, han resultado de utilidad los trabajos de los diversos especialistas citados.

Como conclusiones al presente estudio, se proponen:

a) Al ser la economía un concepto de origen griego, ha resultado provechoso su estudio a partir de la Grecia Antigua. El análisis etimológico ha constituido la base, para el posterior desarrollo dentro del contexto cultural específico.

b) El sistema monetario griego presenta cierta complejidad, por lo que, antes de cualquier consideración de carácter económico, ha sido necesaria una explicación del sistema de pesos y de las distintas variaciones. La equivalencia moderna, en dólares, pese a ser solo una aproximación, ha facilitado la comparación con el mundo actual.

c) La administración financiera ateniense parte de un modelo bélico y esclavista. Esta es la explicación de la presencia de tributos, presupuesto para la guerra y para el pago de esclavos públicos. 
d) Hay distintas concepciones del trabajo asî como diversas ocupaciones. Algunos ejemplos de labor obrera han permitido visualizar a qué se dedicaban los griegos para subsistir. La lista de oficios se podría enriquecer y ser objeto de un estudio léxico.

e) El costo de la vida en la Atenas del siglo V a.C. era elevado: un salario mínimo de cuatro óbolos al día apenas alcanzaba para una persona sola, pero no para una familia de cuatro. Del presupuesto anual, el mayor consumo es en alimento, con un $80 \%$. Aunque, comparativamente, las cifras indican menores costos que en el mundo actual, el poder adquisitivo era, igualmente, significativamente inferior. En suma, detrás de la masiva inversión en el embellecimiento de una ciudad que aún es objeto de admiración debió de existir una población quizás orgullosa, pero cuya vida no ha de haber sido fácil.

\section{REFERENCIAS}

Amemiya, T. 2007. Economy and Economics of Ancient Greece. Routledge. London. $184 \mathrm{p}$.

Bailly, A. 2000. Dictionnaire Grec-Français. Hachette. Paris. 2230 p.

Berenguer, J. 2002. Gramática griega. Bosch. Barcelona. $276 \mathrm{p}$.

Burckhardt, J. 1963. Historia de la cultura griega. Tomo IV. Obras Maestras. Barcelona. 408 p.

Burckhardt, J. 1974. Historia de la cultura griega. Tomo I. Obras Maestras. Barcelona. 408 p.
Real Academia Española. 2013. Diccionario de la Real Academia Española. Madrid, España, 8-11-13, www.rae.es

Flacelière, R. 1959. La vida cotidiana en Grecia en el siglo de Pericles. Hachette. Buenos Aires. $327 \mathrm{p}$.

Hesíodo. 1997. Obras y fragmentos. Traducción de Aurelio Pérez y Alfonso Martínez. Gredos. Madrid. 439 p.

Homero. 2002. Odisea. Traducción de José Manuel Pabón. Gredos. Madrid. 518 p.

Institut national de la statistique et des études économiques. 2013. Convertisseur franceuro : pouvoir d'achat de l'euro et du franc. Paris, France, 1-11-13, www.insee.fr

Jenofonte. 2006. Recuerdos de Sócrates. Económico. Banquete. Apología de Sócrates. Traducción de Juan Zaragoza. Gredos. Madrid. 386 p.

Mirón, M. 2004. Oikos y oikonomia: El análisis de las unidades domésticas de producción y reproducción en el estudio de la Economía Antigua. Gerión. 22 (1): 61-79.

Morkhlom, O. 1982. Some Reflections on the Production and Use of Coinage in Ancient Greece. Historia: Zeitung für Alte Geschichte. 31 (3): 290-305.

Morris, I. 2004. Economic Growth in Ancient Greece. Journal of Institutional and Theoretical Economics 160 (4): 709-742.

Pabón, J. 1999. Diccionario Griego-Español. Vox. Barcelona. $711 \mathrm{p}$.

Petrie, A. 1978. Introducción al estudio de Grecia. FCE. México. 179 p.

Segura, S. 2003. Nuevo diccionario etimológico Latín-Español y de las voces derivadas. Universidad de Deusto. Bilbao. 1251 p. 